Vasile Ene, Ovidius University Constanţa, Romania Current address: 23 August 8717, Jud. Constanţa, Romania

e-mail: ene@523aug.sfes.ro or ene@univ-ovidius.ro

\title{
CONVERGENCE AND APPROXIMATE DIFFERENTIATION
}

\begin{abstract}
The main result of this paper is Theorem 1, which states the following: Let $F, F_{n}:[a, b] \rightarrow \mathbb{R}, n=1,2, \ldots$ be Lebesgue measurable functions such that $\left\{F_{n}\right\}_{n}$ converges pointwise to $F$ on $[a, b]$. If each $F_{n}$ is approximately derivable a.e. on $[a, b],\left\{F_{n}\right\}_{n}$ is uniformly absolutely continuous on a set $P \subset[a, b]$, and $\left\{\left(F_{n}\right)_{a p}^{\prime}\right\}_{n}$ converges in measure to a measurable function $g$, finite a.e. on $[a, b]$, then $F$ is approximately derivable a.e. on $P$ and $F_{a p}^{\prime}(x)=g(x)$ a.e. on $P$. An immediate consequence of this result is the famous theorem of Džvaršeřsili on the passage to the limit for the Denjoy and Denjoy* integrals (see Theorem 47 , p. 40 of [3]). As was pointed out by Bullen in [3] (p. 309), "the $\mathcal{D}^{*}$ integral case of Theorem 47 of [3] was rediscovered by Lee P. Y." (see also Theorem 7.6 of [7]).
\end{abstract}

\section{Preliminaries}

We shall denote the Lebesgue measure of the set $A$ by $m(A)$, whenever $A \subset$ $\mathbb{R}$ is Lebesgue measurable. If $f:[a, b] \rightarrow \mathbb{R}$ and $[\alpha, \beta] \subseteq[a, b]$, then let $\mathcal{O}(F ;[\alpha, \beta]=\sup \{|F(y)-F(x)|: x, y \in[\alpha, \beta]\}$. Let $\mathcal{C}$ denote the class of all continuous functions and $\mathcal{C}_{a p}$ the class of all approximately continuous functions. A function $f: P \rightarrow \mathbb{R}$ is said to satisfy Lusin's condition $(N)$, if $m(F(Z))=0$, whenever $m(Z)=0$. For the definitions of $A C, A C^{*}, V B$ and $V B^{*}$ see [11].

Definition 1. ([11], p. 221). Let $F: P \rightarrow \mathbb{R}$, and $Q \subseteq P$. We denote by $V(F ; Q)$ the upper bound of the numbers $\sum_{i}\left|F\left(b_{i}\right)-F\left(a_{i}\right)\right|$, where $\left\{\left[a_{i}, b_{i}\right]\right\}_{i}$ is any sequence of nonoverlapping closed intervals with endpoints in $Q$. (We may suppose without loss of generality that $\left\{\left[a_{i}, b_{i}\right]\right\}_{i}$ is a finite set.)

Key Words: $U A C, U A C^{*}$, approximate derivative, convergence

Mathematical Reviews subject classification: 26A24, 26A39

Received by the editors January 16, 1997 
Definition 2. Let $E \subseteq[a, b]$. A function $F:[a, b] \rightarrow \mathbb{R}$ is said to be $A C G$ (respectively $A C^{*} G, V B G, V B^{*} G, \mathcal{C} G$ ) on $E$ if there exists a sequence of sets $\left\{E_{n}\right\}$ with $E=\cup_{n} E_{n}$, such that $F$ is $A C$ (respectively $A C^{*}, V B, V B^{*}, \mathcal{C}$ ) on each $E_{n}$. If in addition the sets $E_{n}$ are supposed to be closed we obtain the classes $[A C G],\left[A C^{*} G\right],[V B G],\left[V B^{*} G\right],[\mathcal{C} G]$. Note that $A C G$ and $A C^{*} G$ used here differ from those of [11] (because in our definitions the continuity is not assumed).

\section{Main Theorem}

Lemma 1. Let $P$ be a subset of $[a, b]$ and $F: P \rightarrow \mathbb{R}$ an $A C$ function. Then there exists a function $G: \bar{P} \rightarrow \mathbb{R}, G \in A C$ such that $G_{\mid P}=F$. Moreover, if for $\epsilon>0, \delta_{\epsilon}>0$ is given by the fact that $F \in A C$ on $P$, then $\delta_{\frac{\epsilon}{3}}$ satisfies the definition of $G$ being $A C$ on $\bar{P}$ for $\epsilon$. As a consequence, if $F$ is measurable, then $F$ is approximately derivable a.e. on $P$.

Proof. Let $x_{o} \in \bar{P}$. For $\epsilon>0$ let $\delta_{\epsilon}>0$ be given by the fact that $F \in A C$ on $P$. Then $|F(x)-F(y)|<\epsilon$ whenever $x, y \in\left(x_{o}-\delta_{\epsilon} / 2, x_{o}+\delta_{\epsilon} / 2\right) \cap P$. By the Cauchy criterion, the following limits exist and are finite:

$$
\lim _{x \nearrow x_{o}, x \in P} F(x), \quad \lim _{x \searrow x_{o}, x \in P} F(x), \quad \lim _{x \rightarrow x_{o}, x \in P} F(x),
$$

whenever $x_{o}$ is a left, right or bilateral accumulation point of $P$ respectively. If $x_{o} \in P$ any of the three limits equals $F\left(x_{o}\right)$, provided they exist. Let $G: \bar{P} \rightarrow \mathbb{R}$ be defined by

$$
G(x)= \begin{cases}F(x) & \text { if } x \text { is an isolated point of } P \\ \lim _{\substack{x \succ x_{o} \\ x \in P}} F(x) & \text { if } x \text { is a right accumulation point of } P \\ \lim _{\substack{x \succ x_{o} \\ x \in P}} F(x) & \text { if } x \text { is a left accumulation point of } P .\end{cases}
$$

Let $\left\{\left[a_{i}, b_{i}\right]\right\}, i=1,2, \ldots, n$ be a finite set of closed intervals with endpoints in $\bar{P}, a_{1}<b_{1} \leq a_{2}<b_{2} \leq \cdots \leq a_{n}<b_{n}$, such that $\sum_{i=1}^{n}\left(b_{i}-a_{i}\right)<\delta_{\frac{\epsilon}{3}}$. Let $\mathcal{A}_{1}=\{i: i=$ odd, $i \leq n\}$ and $\mathcal{A}_{2}=\{i: i=$ even, $i \leq n\}$. Then there exists a finite set $\left\{\left[x_{i}, y_{i}\right]\right\}_{i \in \mathcal{A}_{1}}$ of nonoverlapping closed intervals with endpoints in $P$ such that

$$
\left|F\left(x_{i}\right)-G\left(a_{i}\right)\right|<\frac{\epsilon}{6(n+1)}, \quad\left|F\left(y_{i}\right)-G\left(b_{i}\right)\right|<\frac{\epsilon}{6(n+1)}
$$

and

$$
\sum_{i \in \mathcal{A}_{1}}\left(y_{i}-x_{i}\right)<\delta_{\frac{\epsilon}{3}}
$$


It follows that

$$
\begin{gathered}
\sum_{i \in \mathcal{A}_{1}}\left|G\left(b_{i}\right)-G\left(a_{i}\right)\right| \leq \sum_{i \in \mathcal{A}_{1}}\left|G\left(a_{i}\right)-F\left(x_{i}\right)\right|+ \\
+\sum_{i \in \mathcal{A}_{1}}\left|F\left(x_{i}\right)-F\left(y_{i}\right)\right|+\sum_{i \in \mathcal{A}_{1}}\left|F\left(y_{i}\right)-G\left(b_{i}\right)\right|<\frac{\epsilon}{12}+\frac{\epsilon}{3}+\frac{\epsilon}{12}=\frac{\epsilon}{2} .
\end{gathered}
$$

Similarly it follows that

$$
\sum_{i \in \mathcal{A}_{2}}\left|G\left(b_{i}\right)-G\left(a_{i}\right)\right|<\epsilon / 2 .
$$

Therefore $\sum_{i=1}^{n}\left|G\left(b_{i}\right)-G\left(a_{i}\right)\right|<\epsilon$. The last assertion follows from Theorem 4.2 of [11], p. 222 and by the fact that an $A C$ function on a set is $V B$ on that set.

Definition 3. ([3], $p$. 38). Let $P$ be a real set and $F_{n}: P \rightarrow \mathbb{R}, n=1,2, \ldots$

- The sequence $\left\{F_{n}\right\}_{n}$ is said to be $U A C$ on $P$ if it has the following property: for every $\epsilon>0$ there is a $\delta_{\epsilon}>0$ such that $\sum_{k=1}^{m} \mid F_{n}\left(\beta_{k}\right)-$ $F_{n}\left(\alpha_{k}\right) \mid<\epsilon$ for all $n=1,2, \ldots$, whenever $\left\{\left[\alpha_{k}, \beta_{k}\right]\right\}, k=1,2, \ldots, m$ is a finite set of nonoverlapping closed intervals with endpoints in $P$ and $\sum_{k=1}^{m}\left(\beta_{k}-\alpha_{k}\right)<\delta_{\epsilon}$.

- The sequence $\left\{F_{n}\right\}_{n}$ is said to be $U A C G$ on $P$, if $P=\cup P_{k}$ and $\left\{F_{n}\right\}_{n}$ is $U A C$ on each $P_{k}$. If in addition each $P_{k}$ is supposed to be closed, then $\left\{F_{n}\right\}_{n}$ is said to be $[U A C G]$ on $P$.

Remark 1. If $P$ is a closed set, then $[U A C G]$ is in fact Džvaršeǐšvili's condition "UACG" of [3], p. 38 (this follows using the technique of the proof of Theorem 9.1 of [11], p. 233). This fact, for $P=[a, b]$, was pointed out by Bullen (see [3], p. 308).

Corollary 1. Let $P$ be a subset of $[a, b]$ and let $F_{n}: P \rightarrow \mathbb{R}, n=1,2, \ldots$ If $\left\{F_{n}\right\}_{n}$ is $U A C$ on $P$, then there exist $G_{n}: \bar{P} \rightarrow \mathbb{R}, n=1,2, \ldots,\left(G_{n}\right)_{/ P}=F_{n}$, such that $\left\{G_{n}\right\}_{n}$ is $U A C$ on $\bar{P}$. Moreover, if for $\epsilon>0, \delta_{\epsilon}>0$ is given by the fact that $\left\{F_{n}\right\}_{n}$ is $U A C$ on $P$, then $\delta_{\epsilon / 3}$ satisfies the definition of $\left\{G_{n}\right\}_{n}$ being $U A C$ on $\bar{P}$ for $\epsilon$.

Lemma 2. Let $P$ be a closed subset of $[a, b], a, b \in P$ and let $F:[a, b] \rightarrow \mathbb{R}$ be a function which is linear on the closure of each interval contiguous to $P$. Then $V(F ;[a, b])=V(F ; P)$. 
Proof. Clearly $V(F ; P) \leq V(F ;[a, b])$. Therefore we only need to show that $V(F ;[a, b]) \leq V(F ; P)$. Let

$$
\Delta: a=a_{o}<a_{1}<\ldots<a_{m}=b
$$

be a division of $[a, b]$. Let $\Delta_{1}:=\Delta \cup\{$ the endpoints of those intervals contiguous to $P$ which contain points of $\Delta\}$. Suppose that

$$
\Delta_{1}: a=\alpha_{o}<\alpha_{1}<\ldots<\alpha_{n}=b .
$$

Let $\Delta_{2}=\Delta_{1} \cap P$. Suppose that

$$
\Delta_{2}: a=\beta_{1}<\ldots<\beta_{p}=b .
$$

Then

$$
\sum_{i=1}^{m}\left|F\left(a_{i}\right)-F\left(a_{i-1}\right)\right| \leq \sum_{i=1}^{n}\left|F\left(\alpha_{i}\right)-F\left(\alpha_{i-1}\right)\right|=\sum_{i=1}^{p}\left|F\left(\beta_{i}\right)-F\left(\beta_{i-1}\right)\right| .
$$

(The equality follows by the fact that $F$ is linear on the closure of each interval contiguous to $P$.) Therefore $V(F ;[a, b]) \leq V(F ; P)$.

Lemma 3. Let $P$ be a subset of $[a, b]$ and let $F: P \rightarrow \mathbb{R}, F \in A C$. For $\epsilon>0$ let $\delta_{\epsilon}>0$ be given by the fact that $F \in A C$ on $P$. Then there exists a function $\tilde{F}:[a, b] \rightarrow \mathbb{R}, \tilde{F} \in A C$ such that $\tilde{F}_{/ P}=F$ and

$$
(\mathcal{L}) \int_{A}\left|\tilde{F}^{\prime}(t)\right| d t<\epsilon
$$

whenever $A$ is a measurable subset of $\bar{P}$ with $m(A)<\delta_{\epsilon / 6}$.

Proof. For $\epsilon>0$ let $\delta_{\epsilon}>0$ be given by the fact that $F \in A C$ on $P$. Let $c_{o}=\inf (P), d_{o}=\sup (P)$, and let $\left(c_{k}, d_{k}\right), k=1,2, \ldots$ be the intervals contiguous to $\bar{P}$. By Lemma 1 there exists $G: \bar{P} \rightarrow \mathbb{R}$ such that $G \in A C$ on $\bar{P}, G_{/ P}=F$ and for $\epsilon$, the number $\delta_{\frac{\epsilon}{3}}$ is the $\delta$ given by the fact that $G \in A C$ on $\bar{P}$. Let $\tilde{F}:[a, b] \rightarrow \mathbb{R}$ be defined by

$$
\tilde{F}(x)= \begin{cases}G\left(c_{o}\right) & \text { if } x \in\left[a, c_{o}\right] \\ G(x) & \text { if } x \in \bar{P} \\ \text { linearly } & \text { on each }\left[c_{k}, d_{k}\right] \\ G\left(d_{o}\right) & \text { if } x \in\left[d_{o}, b\right] .\end{cases}
$$

Then $\tilde{F} \in A C$ on $[a, b]$. (See for example Theorem 2.11 .1 (xviii) of [4].) Let $A$ be a measurable subset of $\bar{P}$ with $m(A)<\delta_{\epsilon / 6}$. Then there exists a 
sequence $\left\{\left(\alpha_{i}, \beta_{i}\right)\right\}_{i}$ such that $\left(\alpha_{i}, \beta_{i}\right) \cap A \neq \emptyset$ for each $i, A \subset \cup_{i=1}^{\infty}\left(\alpha_{i}, \beta_{i}\right)$ and $\sum_{i=1}^{\infty}\left(\beta_{i}-\alpha_{i}\right)<\delta_{\epsilon / 6}$. Let $a_{i}=\inf \left(\alpha_{i}, \beta_{i}\right) \cap \bar{P}$ and $b_{i}=\sup \left(\alpha_{i}, \beta_{i}\right) \cap \bar{P}$. Then $a_{i}, b_{i} \in \bar{P}$ and

$$
\begin{aligned}
& (\mathcal{L}) \int_{A}\left|\tilde{F}^{\prime}(t)\right| d t \leq \sum_{i=1}^{\infty}(\mathcal{L}) \int_{a_{i}}^{b_{i}}\left|\tilde{F}^{\prime}(t)\right| d t= \\
= & \sum_{i=1}^{\infty} V\left(\tilde{F} ;\left[a_{i}, b_{i}\right]\right)=\sum_{i=1}^{\infty} V\left(G ;\left[a_{i}, b_{i}\right] \cap \bar{P}\right) .
\end{aligned}
$$

(The first equality follows by Theorem 8 of [8], p. 259, and the second equality follows by Lemma 2.)

For each $i$ there exists a division

$$
\Delta_{i}: a_{i}=a_{i, 0}<a_{i, 1} \ldots<a_{i, j_{i}}=b_{i},
$$

with each point in $\bar{P}$ such that

$$
V\left(G ;\left[a_{i}, b_{i}\right] \cap \bar{P}\right)<\frac{\epsilon}{2^{i+1}}+\sum_{k=1}^{j_{i}}\left|G\left(a_{i, k}\right)-G\left(a_{i, k-1}\right)\right| .
$$

By (1) and (2), it follows that

$$
(\mathcal{L}) \int_{A}\left|\tilde{F}^{\prime}(t)\right| d t \leq \sum_{i=1}^{\infty} V\left(G ;\left[a_{i}, b_{i}\right] \cap \bar{P}\right)<\frac{\epsilon}{2}+\frac{\epsilon}{2}=\epsilon .
$$

Corollary 2. (An extension of Lemma 2 of [3], p. 38). Let $P \subseteq[a, b]$ and let $\left\{F_{n}\right\}_{n}$ be a $U A C$ sequence on $P$. For $\epsilon>0$ let $\delta_{\epsilon}>0$ be given by the latter fact. Then there exist $\tilde{F}_{n}:[a, b] \rightarrow \mathbb{R}$ such that $\tilde{F}_{n} \in A C,\left(\tilde{F}_{n}\right)_{/ P}=F_{n}$ and

$$
(\mathcal{L}) \int_{A}\left|\tilde{F}_{n}^{\prime}(t)\right| d t<\epsilon,
$$

for all $n=1,2, \ldots$, whenever $A$ is a measurable subset of $\bar{P}$ with $m(A)<\delta_{\epsilon / 6}$. Proof. Apply Lemma 3 to each $F_{n}$.

Corollary 3. Let $\left\{F_{n}\right\}_{n}$ be an $U A C$ sequence on $[a, b]$, and let $x_{o} \in[a, b]$ such that $\lim _{n \rightarrow \infty} F_{n}\left(x_{o}\right)=\ell \in \mathbb{R}$. Let $g:[a, b] \rightarrow \overline{\mathbb{R}}$ be finite a.e. such that $\left\{F_{n}^{\prime}\right\}_{n}$ converges to $g$ in measure. Then $g$ is Lebesgue integrable on $[a, b]$. Moreover, if $G(x)=\ell+(\mathcal{L}) \int_{x_{o}}^{x} g(t) d t$, then $\left\{F_{n}\right\}_{n}$ converges uniformly to $G$ on $[a, b]$ and $G^{\prime}(x)=g(x)$ a.e. on $[a, b]$. 
Proof. By Corollary 2, the summable functions $F_{n}^{\prime}, n=1,2, \ldots$ have equiabsolutely continuous integrals. (The functions of a family $\mathcal{M}$ of summable functions defined on a set $E$, are said to have equi-absolutely continuous integrals, if for every $\epsilon>0$, there exists a $\delta>0$ such that $\left|\int_{Q} f(x) d x\right|<\epsilon$, $(\forall) f \in \mathcal{M}$, whenever $Q$ is a measurable subset of $E$ with $m(Q)<\delta$; [8], p. 151.) By Vitali's theorem ([8], p. 152), $g$ is Lebesgue integrable on $[a, b]$, and by the proof of the same theorem it follows that

$$
\lim _{n \rightarrow \infty}(\mathcal{L}) \int_{a}^{b}\left|F_{n}^{\prime}(t)-g(t)\right| d t=0 .
$$

For $\epsilon>0$ there exists a positive integer $n_{\epsilon}$ such that

$$
(\mathcal{L}) \int_{a}^{b}\left|F_{n}^{\prime}(t)-g(t)\right| d t<\frac{\epsilon}{2} \text { and }\left|F_{n}\left(x_{o}\right)-\ell\right|<\frac{\epsilon}{2}
$$

whenever $n \geq n_{\epsilon}$. Suppose that $x \geq x_{o}$. Then

$$
\begin{gathered}
\left|F_{n}(x)-G(x)\right|=\left|F_{n}\left(x_{o}\right)+(\mathcal{L}) \int_{x_{o}}^{x} F_{n}^{\prime}(t) d t-\ell-(\mathcal{L}) \int_{x_{o}}^{x} g(t) d t\right| \\
\leq\left|F_{n}\left(x_{o}\right)-\ell\right|+(\mathcal{L}) \int_{x_{o}}^{x}\left|F_{n}^{\prime}(t)-g(t)\right| d t<\frac{\epsilon}{2}+\frac{\epsilon}{2}=\epsilon,
\end{gathered}
$$

whenever $n>n_{\epsilon}$. Similarly, if $x<x_{o}$, then we obtain $\left|F_{n}(x)-G(x)\right|<\epsilon$, whenever $n>n_{\epsilon}$. Therefore $\left\{F_{n}\right\}_{n}$ converges uniformly to $G$ on $[a, b]$.

That $G^{\prime}(x)=g(x)$ a.e. on $[a, b]$ is obvious.

Definition 4. ([3], p. 38). Let $P \subset[a, b]$ and $F_{n}:[a, b] \rightarrow \mathbb{R}, n=1,2, \ldots$

- The sequence $\left\{F_{n}\right\}_{n}$ is said to be $U A C^{*}$ on $P$ if it has the following property: for every $\epsilon>0$ there exists a $\delta_{\epsilon}>0$ such that

$$
\sum_{k=1}^{m} \mathcal{O}\left(F_{n} ;\left[\alpha_{k}, \beta_{k}\right]\right)<\epsilon, \quad n=1,2, \ldots,
$$

whenever $\left\{\left[\alpha_{k}, \beta_{k}\right]\right\}, k=1,2, \ldots, m$ is a set of nonoverlapping closed intervals with endpoints in $P$ and $\sum_{k=1}^{m}\left(\beta_{k}-\alpha_{k}\right)<\delta_{\epsilon}$.

- The sequence $\left\{F_{n}\right\}_{n}$ is said to be $U A C^{*} G$ on $P$, if $P=\cup P_{k}$ and $\left\{F_{n}\right\}_{n}$ is $U A C^{*}$ on each $P_{k}$. If in addition each $P_{k}$ is supposed to be closed, then $\left\{F_{n}\right\}_{n}$ is said to be $\left[U A C^{*} G\right]$ on $P$. 
Remark 2. If $P$ is a closed set, then $\left[U A C^{*} G\right]$ is in fact Džvaršeǐšvili's condition " $U A C G^{*}$ " of [3], p. 38. (This follows using the technique of the proof of Theorem 9.1 of [11], p. 233.) If $P=[a, b]$ and each $F_{n}$ is supposed to be continuous on $[a, b]$, then $\left[U A C^{*} G\right]$ on $[a, b]$ is identical with P. Y. Lee's Definition 7.4, (ii) of [7], p. 39.

Lemma 4. Let $P \subset[a, b]$ and $F, F_{n}:[a, b] \rightarrow \mathbb{R}, n=1,2, \ldots$

(i) If $\left\{F_{n}\right\}_{n}$ is $U A C$ on $P$ and converges pointwise to $F$ on $P$, then $F \in A C$ on $P$.

(ii) If $\left\{F_{n}\right\}_{n}$ is $U A C^{*}$ on $P$ and converges pointwise to $F$ on $[a, b]$, then $F \in A C^{*}$ on $P$.

Proof. (i) For $\epsilon>0$ let $\delta_{\epsilon}>0$ be given by the fact that $\left\{F_{n}\right\}_{n}$ is $U A C$ on $P$. Let $\left\{\left[a_{i}, b_{i}\right]\right\}, i=1,2, \ldots, m$ be a set of nonoverlapping closed intervals with endpoints in $P$ such that $\sum_{i=1}^{m}\left(b_{i}-a_{i}\right)<\delta_{\epsilon / 2}$. Then for each $n=1,2, \ldots$ we have

$$
\sum_{i=1}^{m}\left|F_{n}\left(b_{i}\right)-F_{n}\left(a_{i}\right)\right|<\frac{\epsilon}{2} .
$$

Passing to the limit, we obtain that

$$
\sum_{i=1}^{m}\left|F\left(b_{i}\right)-F\left(a_{i}\right)\right| \leq \frac{\epsilon}{2}<\epsilon .
$$

Hence $F \in A C$ on $P$.

(ii) For $\epsilon>0$ let $\delta_{\epsilon}>0$ be given by the fact that $\left\{F_{n}\right\}_{n}$ is $U A C^{*}$ on $P$. Let $\left\{\left[a_{i}, b_{i}\right]\right\}, i=1,2, \ldots, m$, be a set of nonoverlapping closed intervals with endpoints in $P$, such that $\sum_{i=1}^{m}\left(b_{i}-a_{i}\right)<\delta_{\epsilon / 3}$. Then for each $n=1,2, \ldots$

$$
\sum_{i=1}^{m} \mathcal{O}\left(F_{n} ;\left[a_{i}, b_{i}\right]\right)<\frac{\epsilon}{3} .
$$

Since $\left\{F_{n}\right\}_{n}$ converges pointwise to $F$ on $[a, b]$, it follows that for each $i=$ $1,2, \ldots, m$ we have $\mathcal{O}\left(F ;\left[a_{i}, b_{i}\right]\right) \leq \epsilon<+\infty$. Thus, for each $i=1,2, \ldots, m$, there exists $\left[\alpha_{i}, \beta_{i}\right] \subseteq\left[a_{i}, b_{i}\right]$ such that

$$
\mathcal{O}\left(F ;\left[a_{i}, b_{i}\right]\right)<\left|F\left(\beta_{i}\right)-F\left(\alpha_{i}\right)\right|+\frac{\epsilon}{2^{i}} .
$$

Let $n$ be a positive integer such that

$$
\left|F_{n}\left(\alpha_{i}\right)-F\left(\alpha_{i}\right)\right|<\frac{\epsilon}{6 m} \text { and }\left|F_{n}\left(\beta_{i}\right)-F\left(\beta_{i}\right)\right|<\frac{\epsilon}{6 m} \quad i=1,2, \ldots, m .
$$


(This is possible because $\left\{F_{n}\right\}_{n}$ converges pointwise to $F$ on $\left.[a, b].\right)$ Then

$$
\begin{aligned}
& \sum_{i=1}^{m} \mathcal{O}\left(F ;\left[a_{i}, b_{i}\right]\right)<\frac{\epsilon}{3}+\sum_{i=1}^{m}\left|F\left(\beta_{i}\right)-F\left(\alpha_{i}\right)\right| \leq \frac{\epsilon}{3}+\sum_{i=1}^{m}\left|F\left(\beta_{i}\right)-F_{n}\left(\beta_{i}\right)\right| \\
& +\sum_{i=1}^{m}\left|F_{n}\left(\beta_{i}\right)-F_{n}\left(\alpha_{i}\right)\right|+\sum_{i=1}^{m}\left|F_{n}\left(\alpha_{i}\right)-F\left(\alpha_{i}\right)\right|<\frac{\epsilon}{3}+\frac{\epsilon}{6}+\frac{\epsilon}{3}+\frac{\epsilon}{6}=\epsilon .
\end{aligned}
$$

Hence $F \in A C^{*}$ on $P$.

Lemma 5. Let $P$ be a closed subset of $[a, b], a, b \in P$, and let $F, F_{n}:[a, b] \rightarrow$ $\mathbb{R}, n=1,2, \ldots$, be such that $F$ and each $F_{n}$ are linear on the closure of each interval contiguous to $P$. If $\left\{F_{n}\right\}_{n}$ is $U A C$ on $P$ and converges pointwise to $F$ on $P$, then $\left\{F_{n}\right\}_{n}$ is $U A C$ on $[a, b]$ and $F \in A C$ on $[a, b]$. Consequently $F$ and $F_{n}$ are derivable a.e. on $[a, b]$. Moreover, if $\left\{F_{n}^{\prime}\right\}_{n}$ converges in measure to an a.e. finite function $g$ on $P$, then $F^{\prime}(x)=g(x)$ a.e. on $P$.

Proof. We consider for example the case when the set of all intervals contiguous to $P$ is infinite. Let $\left\{\left(c_{k}, d_{k}\right)\right\}, k=1,2, \ldots$ be the intervals contiguous to $P$. Since $\left\{F_{n}\right\}_{n}$ converges pointwise to $F$ on $P$, it follows that $\left\{F_{n}\right\}_{n}$ converges pointwise to $F$ on $[a, b]$. For $\epsilon>0$ let $\delta_{\epsilon}>0$ be given by the fact that $\left\{F_{n}\right\}_{n}$ is $U A C$ on $P$. Let $k_{\epsilon}$ be a positive integer such that $\sum_{k=1+k_{\epsilon}}^{\infty}\left(d_{k}-c_{k}\right)<\delta_{\epsilon}$. Since $\left\{F_{n}\right\}_{n}$ converges pointwise to $F$ on $P$, there exists a positive integer $n_{\epsilon}$ such that

$$
\frac{\left|F_{n}\left(d_{k}\right)-F_{n}\left(c_{k}\right)\right|}{d_{k}-c_{k}}<1+\frac{\left|F\left(d_{k}\right)-F\left(c_{k}\right)\right|}{d_{k}-c_{k}} \quad \text { for each } k=1,2, \ldots, k_{\epsilon},
$$

whenever $n \geq 1+n_{\epsilon}$. Let

$$
\begin{gathered}
M_{\epsilon}=1+\max _{\substack{n=1, \ldots, n_{\epsilon} \\
k=1, \ldots, k_{\epsilon}}}\left\{\frac{\left|F_{n}\left(d_{k}\right)-F_{n}\left(c_{k}\right)\right|}{d_{k}-c_{k}}, \frac{\left|F\left(d_{k}\right)-F\left(c_{k}\right)\right|}{d_{k}-c_{k}}\right\}, \\
\eta_{\epsilon}=\min \left\{\frac{\epsilon}{M_{\epsilon}}, \delta_{\epsilon}\right\} .
\end{gathered}
$$

Let $\left\{\left[\alpha_{i}, \beta_{i}\right]\right\}, i=1,2, \ldots, m$ be a finite set of nonoverlapping closed subintervals of $[a, b]$ with $\sum_{i=1}^{m}\left(\beta_{i}-\alpha_{i}\right)<\eta_{\epsilon}$. If $\left(\alpha_{i}, \beta_{i}\right) \cap P \neq \emptyset$, let $\alpha_{i}^{\prime}=\inf \left(\left(\alpha_{i}, \beta_{i}\right) \cap\right.$ $P)$ and $\beta_{i}^{\prime}=\sup \left(\left(\alpha_{i}, \beta_{i}\right) \cap P\right)$. Then $\left[\alpha_{i}, \beta_{i}\right]=\left[\alpha_{i}, \alpha_{i}^{\prime}\right] \cup\left[\alpha_{i}^{\prime}, \beta_{i}^{\prime}\right] \cup\left[\beta_{i}^{\prime}, \beta_{i}\right]$. Therefore $\cup_{i=1}^{m}\left[\alpha_{i}, \beta_{i}\right]$ can also be written as the union of a finite set $\left\{\left[a_{j}, b_{j}\right]\right\}$, $j=1,2, \ldots, p, p \leq 3 m$, of nonoverlapping nondegenerate closed intervals such that either $\left[a_{j}, b_{j}\right] \subseteq\left[c_{k}, d_{k}\right]$ for some $k$, or both $a_{j}$ and $b_{j}$ belong to $P$. Let 


$$
\begin{aligned}
& \mathcal{A}_{1}=\left\{j: a_{j}, b_{j} \in P\right\} ; \\
& \mathcal{A}_{2}=\left\{j:\left[a_{j}, b_{j}\right] \subset \cup_{k=1+k_{\epsilon}}^{\infty}\left[c_{k}, d_{k}\right]\right\} ; \\
& \mathcal{A}_{3}=\left\{j:\left[a_{j}, b_{j}\right] \subset \cup_{k=1}^{k_{\epsilon}}\left[c_{k}, d_{k}\right]\right\} .
\end{aligned}
$$

By (5) we have

$$
\sum_{j \in \mathcal{A}_{1}}\left|F_{n}\left(b_{j}\right)-F_{n}\left(a_{j}\right)\right|<\epsilon, \quad \text { for each } n .
$$

Because $F_{n}$ is linear on each $\left[c_{k}, d_{k}\right]$ it follows that

$$
\sum_{j \in \mathcal{A}_{2}}\left|F_{n}\left(b_{j}\right)-F_{n}\left(a_{j}\right)\right| \leq \sum_{k=1+k_{\epsilon}}^{\infty}\left|F_{n}\left(d_{k}\right)-F_{n}\left(c_{k}\right)\right|<\epsilon .
$$

Let $i \in \mathcal{A}_{3}$ and $k \leq k_{\epsilon}$ such that $\left[a_{i}, b_{i}\right] \subset\left[c_{k}, d_{k}\right]$. If $n \geq 1+n_{\epsilon}$, then by (3) and (4) it follows that

$$
\frac{\left|F_{n}\left(b_{j}\right)-F_{n}\left(a_{j}\right)\right|}{b_{j}-a_{j}}=\frac{\left|F_{n}\left(d_{k}\right)-F_{n}\left(c_{k}\right)\right|}{d_{k}-c_{k}}<1+\frac{\left|F\left(d_{k}\right)-F\left(c_{k}\right)\right|}{d_{k}-c_{k}}<M_{\epsilon} .
$$

If $n \leq n_{\epsilon}$, then by (4) it follows that

$$
\frac{\left|F_{n}\left(b_{j}\right)-F_{n}\left(a_{j}\right)\right|}{b_{j}-a_{j}}=\frac{\left|F_{n}\left(d_{k}\right)-F_{n}\left(c_{k}\right)\right|}{d_{k}-c_{k}}<M_{\epsilon} .
$$

By (5), for each $n$ we have

$$
\sum_{j \in \mathcal{A}_{3}}\left|F_{n}\left(b_{j}\right)-F_{n}\left(a_{j}\right)\right|<M_{\epsilon} \cdot \sum_{j \in \mathcal{A}_{3}}\left(b_{j}-a_{j}\right)<M_{\epsilon} \cdot \frac{\epsilon}{M_{\epsilon}}=\epsilon .
$$

By (6), (7) and (8) it follows that

$$
\sum_{i=1}^{m}\left|F_{n}\left(\beta_{i}\right)-F_{n}\left(\alpha_{i}\right)\right| \leq \sum_{j=1}^{p}\left|F\left(b_{j}\right)-F\left(a_{j}\right)\right|<3 \epsilon,
$$

for each $n$. Therefore $\left\{F_{n}\right\}_{n}$ is $U A C$ on $[a, b]$. That $F \in A C$ on $[a, b]$ follows by Lemma 4 , (i). Clearly $F$ and $F_{n}$ are derivable a.e. on $[a, b]$.

We prove the second part. Let $x \in\left(c_{k}, d_{k}\right)$ for some $k$. Then

$$
F_{n}^{\prime}(x)=\frac{F_{n}\left(d_{k}\right)-F_{n}\left(c_{k}\right)}{d_{k}-c_{k}} \longrightarrow \frac{F\left(d_{k}\right)-F\left(c_{k}\right)}{d_{k}-c_{k}} \text { if } n \rightarrow \infty .
$$


Let

$$
g_{o}(x)= \begin{cases}g(x) & \text { if } x \in P \\ \frac{F\left(d_{k}\right)-F\left(c_{k}\right)}{d_{k}-c_{k}} & \text { if } x \in\left(c_{k}, d_{k}\right) \text { for each } k \\ 0 & \text { if } x \in\left[a, c_{o}\right] \cup\left[d_{o}, b\right]\end{cases}
$$

Since $\left\{F_{n}^{\prime}\right\}_{n}$ converges in measure to $g$ on $P$, it follows that it also converges in measure to $g_{o}$ on $[a, b]$. By Corollary $3, g_{o}$ is Lebesgue integrable on $[a, b]$ and $\left\{F_{n}\right\}_{n}$ converges uniformly to $G$ on $[a, b]$, where $G(x)=F(a)+(\mathcal{L}) \int_{a}^{x} g_{o}(t) d t$. Since $\left\{F_{n}\right\}_{n}$ converges to $F$ on $[a, b]$ it follows that $F=G$ on $[a, b]$. Hence $F^{\prime}(x)=G^{\prime}(x)=g_{o}(x)$ a.e. on $[a, b]$. Therefore $F^{\prime}(x)=g(x)$ a.e. on $P$.

Remark 3. In Lemma 5, the condition " $\left\{F_{n}\right\}_{n}$ converges pointwise to $F$ : $P \rightarrow \mathbb{R}$ on $P$ " is essential. Indeed, let $P=[0,1 / 3] \cup[2 / 3,1]$ and let $F_{n}: P \rightarrow \mathbb{R}$,

$$
F_{n}(x)= \begin{cases}0 & \text { if } x \in[0,1 / 3] \\ n & \text { if } x \in[2 / 3,1]\end{cases}
$$

For $\epsilon>0$ and $\delta_{\epsilon}<1 / 3$ we see easily that $\left\{F_{n}\right\}_{n}$ is $U A C$ on $P$, but $\left\{\tilde{F}_{n}\right\}_{n}$ is not $U A C$ on $[0,1]$.

Corollary 4. Let $P$ be a closed subset of $[a, b]$. Let $F, F_{n}:[a, b] \rightarrow \mathbb{R}, n=$ $1,2, \ldots$

(I) Suppose that $\left\{F_{n}\right\}_{n}$ is $U A C$ on $P$ and converges pointwise to $F$ on $P$. We have:

(i) $F \in A C$ on $P$. Consequently $F$ and $F_{n}$ are approximately derivable a.e. on $P$;

(ii) If $\left\{\left(F_{n}\right)_{a p}^{\prime}\right\}_{n}$ converges in measure to an almost everywhere finite function $g$ on $P$, then $F_{a p}^{\prime}(x)=g(x)$ a.e. on $P$.

(II) Suppose that $\left\{F_{n}\right\}_{n}$ is $U A C^{*}$ on $P$ and converges pointwise to $F$ on $[a, b]$. We have:

(i) $F \in A C^{*}$ on $P$. Consequently $F$ and $F_{n}$ are derivable a.e. on $P$.

(ii) If $\left\{F_{n}^{\prime}\right\}_{n}$ converges in measure to an almost everywhere finite function $g$ on $P$, then $F^{\prime}(x)=g(x)$ a.e. on $P$.

Proof. (I) (i) This follows by Lemma 4, (i).

(ii) We may suppose without loss of generality that $a, b \in P$ and that the 
set of all intervals contiguous to $P$ is infinite. Let $\left\{\left(c_{k}, d_{k}\right)\right\}_{k}$ be the intervals contiguous to $P$. Let $\tilde{F}, \tilde{F}_{n}:[a, b] \rightarrow \mathbb{R}$ be defined by

$$
\tilde{F}(x)=\left\{\begin{array}{ll}
F(x) & \text { if } x \in P \\
\text { linear } & \text { on each }\left[c_{k}, d_{k}\right]
\end{array} \text { and } \tilde{F}_{n}(x)= \begin{cases}F_{n}(x) & \text { if } x \in P \\
\text { linear } & \text { on each }\left[c_{k}, d_{k}\right]\end{cases}\right.
$$

Clearly $\tilde{F}_{n}$ converges pointwise to $\tilde{F}$ on $[a, b]$. By Lemma 5 it follows that $\left\{\tilde{F}_{n}\right\}_{n}$ is $U A C$ on $[a, b]$ and $\tilde{F} \in A C$ on $[a, b]$. Clearly $\tilde{F}_{n}^{\prime}(x)=\left(F_{n}\right)_{a p}^{\prime}(x)$ and $\tilde{F}^{\prime}(x)=F_{a p}^{\prime}(x)$ a.e. on $P$. By hypothesis $\left\{\tilde{F}_{n}^{\prime}\right\}_{n}$ converges in measure to an almost everywhere finite function $g$ on $P$, so, by Lemma $5, \tilde{F}^{\prime}(x)=g(x)$ a.e. on $P$. Hence $F_{a p}^{\prime}(x)=g(x)$ a.e. on $P$.

(II) (i) follows by Lemma 4, (ii); (ii) follows by (II) (i) and (I) (ii).

Remark 4. The condition " $\left\{F_{n}\right\}_{n}$ is $U A C$ on $P$ " in Corollary 4, (I) is essential (see Example 2). The condition " $\left\{F_{n}\right\}_{n}$ is $U A C^{*}$ on $P$ " in Corollary 4 , (II) is essential. It cannot be replaced by " $\left\{f_{n}\right\}_{n}$ is $U A C$ on $P$ " (see Example 1).

Theorem 1. Let $P \subseteq[a, b]$ and let $F, F_{n}:[a, b] \rightarrow \mathbb{R}, n=1,2, \ldots$, be measurable functions such that $\left\{F_{n}\right\}_{n}$ converges pointwise to $F$ on $[a, b]$.

(i) Suppose that $F_{n}$ is approximately derivable a.e. on $[a, b],\left\{F_{n}\right\}_{n}$ is $U A C G$ on $P$, and $\left\{\left(F_{n}\right)_{a p}^{\prime}\right\}_{n}$ converges in measure to a measurable function $g$, finite a.e. on $[a, b]$. Then $F$ is approximately derivable a.e. on $P$ and $F_{a p}^{\prime}(x)=g(x)$ a.e. on $P$.

(ii) Suppose that $F_{n}$ is derivable a.e. on $[a, b],\left\{F_{n}\right\}_{n}$ is $U A C^{*} G$ on $P$, and $\left\{F_{n}^{\prime}\right\}_{n}$ converges in measure to a measurable function $g$, finite a.e. on $[a, b]$. Then $F$ is derivable a.e. on $P$ and $F^{\prime}(x)=g(x)$ a.e. on $P$.

Proof. (i) We may suppose without loss of generality that $\left\{F_{n}\right\}_{n}$ is $U A C$ on $P$. By Corollary 1 there exists $G_{n}: \bar{P} \rightarrow \mathbb{R}$ such that $\left\{G_{n}\right\}_{n}$ is $U A C$ on $\bar{P}$ and $\left(G_{n}\right)_{/ P}=F_{n}$ for each $n$. Let $P_{n}=\left\{x \in \bar{P}: F_{n}(x)=G_{n}(x)\right\}$. Then each $P_{n}$ is a Lebesgue measurable set which contains $P$. Let $Q=\cap_{n=1}^{\infty} P_{n}$. Then $Q$ is a Lebesgue measurable subset of $[a, b]$ which contains $P$. It follows that $Q$ can be written as the union of an ascending sequence of closed sets $\left\{Q_{i}\right\}_{i}$ and a null set $Z$. For each $i,\left\{F_{n}\right\}_{n}$ is $U A C$ on $Q_{i}$. By hypothesis and Corollary 4, (I), it follows that $F$ is approximately derivable a.e. on $Q_{i}$ and $F_{a p}^{\prime}(x)=g(x)$ a.e. on $Q_{i}$, for each $i$. Hence $F_{a p}^{\prime}(x)=g(x)$ a.e. on $P$.

(ii) We may suppose without loss of generality that $\left\{F_{n}\right\}_{n}$ is $U A C^{*}$ on $P$. By Lemma 4, (ii) it follows that $F \in A C^{*}$ on $P$. Therefore $F$ is derivable a.e. on $P$. Now the proof follows by (i). 
Remark 5. The condition " $\left\{F_{n}\right\}_{n}$ is $U A C G$ on $P$ " in Theorem 1, (i) is essential (see Example 2). The condition " $\left\{F_{n}\right\}_{n}$ is $U A C^{*} G$ on $P$ " in Theorem 1 , (ii) is also essential. It cannot be replaced by " $\left\{F_{n}\right\}_{n}$ is $U A C$ on $P$ " (see Example 1).

Remark 6. In Corollary 3, Lemma 5, Corollary 4 and Theorem 1 the condition "converges in measure" may be replaced by "converges a.e." (see for example Lebesgue's theorem of [8], p. 95).

\section{Applications of the Main Theorem to Some Integrals, More General Than $\mathcal{D}$ and $\mathcal{D}^{*}$}

Definition 5. Let $\mathcal{M}([a, b])=\{F:[a, b] \rightarrow \mathbb{R}: F$ is a Lebesgue measurable function on $[a, b]\}$. Let $L_{1}, L_{2}, L_{3}$ and $L_{4}$ be linear subspaces of $\mathcal{M}([a, b])$ with the following properties:

1) If $F \in A C G \cap L_{1}$ on $[a, b]$ and $F_{a p}^{\prime}=0$ a.e. on $[a, b]$, then $F$ is a constant function on $[a, b]$.

2) If $F \in[A C G] \cap L_{2}$ on $[a, b]$ and $F_{a p}^{\prime}=0$ a.e. on $[a, b]$, then $F$ is a constant function on $[a, b]$.

3) If $F \in A C^{*} G \cap L_{3}$ on $[a, b]$ and $F^{\prime}=0$ a.e. on $[a, b]$, then $F$ is a constant function on $[a, b]$.

4) If $F \in\left[A C^{*} G\right] \cap L_{2}$ on $[a, b]$ and $F^{\prime}=0$ a.e. on $[a, b]$, then $F$ is a constant function on $[a, b]$.

Remark 7. Clearly there are more subspaces of type $L_{2}$ than of type $L_{1}$, and there are more subspaces of type $L_{4}$ than of type $L_{3}$.

Definition 6. Let $f:[a, b] \rightarrow \overline{\mathbb{R}}$

- $f$ is said to be $L_{1} \mathcal{D}$ (respectively $\left[L_{2} \mathcal{D}\right]$ ) integrable on $[a, b]$, if there exists $F:[a, b] \rightarrow \mathbb{R}$ such that $F \in A C G \cap L_{1}$ (respectively $F \in[A C G] \cap L_{2}$ ) on $[a, b]$, and $F_{a p}^{\prime}(x)=f(x)$ a.e. on $[a, b]$.

- $f$ is said to be $L_{3} \mathcal{D}^{*}$ (respectively $\left[L_{4} \mathcal{D}^{*}\right]$ ) integrable on $[a, b]$, if there exists $F:[a, b] \rightarrow \mathbb{R}$ such that $F \in A C^{*} G \cap L_{3}$ (respectively $F \in$ $\left.\left[A C^{*} G\right] \cap L_{4}\right)$ on $[a, b]$, and $F^{\prime}(x)=f(x)$ a.e. on $[a, b]$.

We shall say that the function $F$ is an indefinite $L_{1} \mathcal{D}$ (respectively $\left[L_{2} \mathcal{D}\right]$, $\left.L_{3} \mathcal{D}^{*},\left[L_{4} \mathcal{D}^{*}\right]\right)$ integral of $f(x)$. Its increment $F(b)-F(a)$ is called the definite $L_{1} \mathcal{D}$ (respectively $\left[L_{2} \mathcal{D}\right], L_{3} \mathcal{D}^{*},\left[L_{4} \mathcal{D}^{*}\right]$ ) integral of $f(x)$, and we denote it by $L_{1} \mathcal{D} \int_{a}^{b} f(t) d t$ (respectively $\left.\left[L_{2} \mathcal{D}\right] \int_{a}^{b} f(t) d t, L_{3} \mathcal{D}^{*} \int_{a}^{b} f(t) d t,\left[L_{4} \mathcal{D}^{*}\right] \int_{a}^{b} f(t) d t\right)$. 


\section{Remark 8.}

- If $L_{1}=L_{2}=L_{3}=L_{4}=\mathcal{C}$, then $\mathcal{C D}=[\mathcal{C D}]=\mathcal{D}$ (the wide Denjoy integral), and $\mathcal{C D}^{*}=\left[\mathcal{C D}^{*}\right]=\mathcal{D}^{*}$ (the Denjoy* integral).

- If $L_{1}=L_{2}=L_{3}=L_{4}=\mathcal{C}_{a p}$, then $\left[\mathcal{C}_{a p} \mathcal{D}\right]$ is the $\beta$-Ridder integral (see Definition 7 of [9], p. 148), which is also called the $A D$-integral of Kubota (see [5], p. 715).

- We have

$$
\begin{gathered}
A C^{*} G \cap \mathcal{C}_{a p} \subset V B^{*} G \cap \mathcal{C}_{a p} \cap(N)=\left[V B^{*} G\right] \cap \mathcal{C}_{a p} \cap[\mathcal{C} G] \cap(N)= \\
=\left[V B^{*} G\right] \cap[A C G] \cap \mathcal{C}_{a p} \subset\left[A C^{*} G\right] \cap \mathcal{C}_{a p} \text { on }[a, b] .
\end{gathered}
$$

For the first equality see Theorem 2.10.3, (vi) of [4] and use the fact that a $\mathcal{C}_{a p}$ function is a Darboux function on an interval. The second equality follows by the Banach-Zarecki Theorem ([11], p. 227). The last inclusion follows by Theorem 2.12.1, (ii) of [4]. Therefore

$$
A C^{*} G \cap \mathcal{C}_{a p}=\left[A C^{*} G\right] \cap \mathcal{C}_{a p},
$$

so

$$
\mathcal{C}_{a p} \mathcal{D}^{*}=\left[\mathcal{C}_{a p} \mathcal{D}^{*}\right]=\alpha-\text { Ridder integral }
$$

(for the $\alpha$-Ridder integral see Definition 2 of [9], p. 138).

- The $L D G$ integrals, introduced by C. M. Lee [6] are $\left[L_{2} \mathcal{D}\right]$-type integrals.

- (Question) Does the $\mathcal{C}_{a p} \mathcal{D}$ integral strictly extend the $\left[\mathcal{C}_{a p} \mathcal{D}\right]$ integral?

Theorem 2. Let $\left\{f_{n}\right\}_{n} \subset L_{1} \mathcal{D}$ (respectively $\left[L_{2} \mathcal{D}\right]$ ) on $[a, b]$ such that

$$
\lim _{n \rightarrow \infty} f_{n} \rightarrow f \text {, a.e. on }[a, b] \text {. }
$$

For each positive integer $n$, let $F_{n}$ be the indefinite $L_{1} \mathcal{D}$ (respectively $\left[L_{2} \mathcal{D}\right]$ ) integral of $f_{n}$. Suppose that $\left\{F_{n}\right\}_{n}$ converges pointwise to $F$ on $[a, b], F \in L_{1}$ (respectively $\left.L_{2}\right)$. If $\left\{F_{n}\right\}_{n} \in U A C G$ (respectively $[U A C G]$ ) on $[a, b]$, then $f \in L_{1} \mathcal{D}$ (respectively $\left[L_{2} \mathcal{D}\right]$ ) on $[a, b]$ and

$$
\lim _{n \rightarrow \infty} L_{1} \mathcal{D} \int_{a}^{b} f_{n}(t) d t=L_{1} \mathcal{D} \int_{a}^{b} f(t) d t
$$

(respectively

$$
\left.\lim _{n \rightarrow \infty}\left[L_{2} \mathcal{D}\right] \int_{a}^{b} f_{n}(t) d t=\left[L_{2} \mathcal{D}\right] \int_{a}^{b} f(t) d t\right)
$$


Proof. See Lemma 4, (i) and Theorem 1, (i).

Theorem 3. Let $\left\{f_{n}\right\}_{n} \subset L_{3} \mathcal{D}^{*}$ (respectively $\left[L_{4} \mathcal{D}^{*}\right]$ ) on $[a, b]$ such that

$$
\lim _{n \rightarrow \infty} f_{n} \rightarrow f \text {, a.e. on }[a, b] .
$$

For each $n$, let $F_{n}$ be the indefinite $L_{2} \mathcal{D}^{*}$ (respectively $\left[L_{4} \mathcal{D}^{*}\right]$ ) integral of $f_{n}$. Suppose that $\left\{F_{n}\right\}_{n}$ converges pointwise to $F$ on $[a, b], F \in L_{3}$ (respectively $L_{4}$ ). If $\left\{F_{n}\right\}_{n} \in U A C^{*} G$ (respectively $\left[U A C^{*} G\right]$ ) on $[a, b]$, then $f \in L_{3} \mathcal{D}^{*}$ (respectively $\left[L_{4} \mathcal{D}^{*}\right]$ ) on $[a, b]$ and

$$
\lim _{n \rightarrow \infty} L_{3} \mathcal{D}^{*} \int_{a}^{b} f_{n}(t) d t=L_{3} \mathcal{D}^{*} \int_{a}^{b} f(t) d t
$$

(respectively

$$
\left.\lim _{n \rightarrow \infty}\left[L_{4} \mathcal{D}^{*}\right] \int_{a}^{b} f_{n}(t) d t=\left[L_{4} \mathcal{D}^{*}\right] \int_{a}^{b} f(t) d t\right) .
$$

Proof. See Lemma 4, (ii) and Theorem 1, (ii).

Remark 9. Suppose that $L_{1}, L_{2}, L_{3}$ and $L_{4}$ are closed under uniform convergence. Then the condition " $\left\{F_{n}\right\}_{n}$ converges pointwise to $F$ on $[a, b], F \in L_{1}$ (respectively $\left.L_{2}\right)$ " in Theorem 2 may be replaced with the condition " $\left\{F_{n}\right\}_{n}$ converges uniformly to $F$ on $[a, b]$ ". Similarly the condition " $\left\{F_{n}\right\}_{n}$ converges pointwise to $F$ on $[a, b], F \in L_{3}$ (respectively $L_{4}$ )" in Theorem 3 may be replaced with the condition " $\left\{F_{n}\right\}_{n}$ converges uniformly to $F$ on $[a, b]$ ".

Note that Theorem 2 contains Theorem 47, a) of [3] and Theorem 3 contains Theorem 47, b) of [3] (in fact Theorem 47, b) is identical with L. P. Yee's Theorem 7.6 of [7]). Theorem 3 also contains L. P. Yee's Corollary 7.7 of [7].

\section{Sequences of Approximately Derivable Functions on an Interval}

We recall the following classical theorems.

Theorem A. ([10], p. 140). Let $\left\{f_{n}\right\}_{n}$ be a sequence of differentiable functions on $[a, b]$, such that $\left\{f_{n}\left(x_{o}\right)\right\}_{n}$ converges for some point $x_{o}$ on $[a, b]$. If $\left\{f_{n}^{\prime}\right\}_{n}$ converges uniformly on $[a, b]$ to $g$, then $\left\{f_{n}\right\}_{n}$ converges uniformly on $[a, b]$ to a function $f$, and $f^{\prime}(x)=g(x)$ on $[a, b]$. 
Remark 10. If in Theorem A, the condition " $\left\{f_{n}^{\prime}\right\}_{n}$ converges uniformly on $[a, b]$ to $g$ " is replaced by " $\left\{f_{n}^{\prime}\right\}_{n}$ converges pointwise on $[a, b]$ to $g$ ", then, even if $\left\{f_{n}\right\}_{n}$ converges uniformly to $f$ on $[a, b]$, it may happen that $f^{\prime}(x)$ does not exist (finite or infinite) on a perfect set of positive measure as close as we want to $b-a$. It follows that $f^{\prime}(x) \neq g(x)$ on a set of positive measure (see Example $1)$.

Theorem B. ([2], p. 44). Let $\left\{f_{n}\right\}_{n}$ be a sequence of approximately differentiable functions on $[a, b]$, such that $\left\{f_{n}\left(x_{o}\right)\right\}_{n}$ converges for some point $x_{o}$ on $[a, b]$. If $\left\{\left(f_{n}\right)_{a p}^{\prime}\right\}_{n}$ converges uniformly on $[a, b]$ to $g$, then $\left\{f_{n}\right\}_{n}$ converges uniformly on $[a, b]$ to a function $f$, and $f_{a p}^{\prime}(x)=g(x)$ on $[a, b]$.

Proof. We follow the proof of [2], p. 44. Since

$$
\left(f_{n}\right)_{a p}^{\prime} \longrightarrow g[\text { unif }] \text { on }[a, b]
$$

it follows that there exists a positive integer $n_{1}$ such that

$$
\left|\left(f_{n}\right)_{a p}^{\prime}(x)-\left(f_{n_{1}}\right)_{a p}^{\prime}(x)\right|<1, \quad(\forall) n \geq n_{1} .
$$

By Tolstoff's Theorem ([1], p. 175) it follows that $f_{n}-f_{n_{1}}$ is a Lipschitz function, and by the Khintchine-Mišik Theorem ([12], p. 139 or [1], Theorem 2.4 , p. 155) we have

$$
\left(f_{n}\right)_{a p}^{\prime}(x)-\left(f_{n_{1}}\right)_{a p}^{\prime}(x)=\left(f_{n}-f_{n_{1}}\right)^{\prime}(x) \quad \text { on } \quad[a, b], \quad(\forall) n \geq n_{1} .
$$

Hence

$$
\left(f_{n}-f_{n_{1}}\right)^{\prime} \longrightarrow g-\left(f_{n_{1}}\right)_{a p}^{\prime}[\text { unif }] \text { on }[a, b] .
$$

By Theorem A

$$
f_{n}-f_{n_{1}} \longrightarrow f-f_{n_{1}}[\text { unif }] \text { on }[a, b] \text { for some } f
$$

and

$$
\left(f-f_{n_{1}}\right)^{\prime}(x)=g(x)-\left(f_{n_{1}}\right)_{a p}^{\prime}(x) \text { on }[a, b]
$$

Therefore

$$
\left(f_{n}\right)_{a p}^{\prime}(x)=\left(f_{n_{1}}\right)_{a p}^{\prime}(x)+\left(f-f_{n_{1}}\right)^{\prime}(x)=g(x) \text { on }[a, b] .
$$

Remark 11. If in Theorem A the condition " $\left\{f_{n}^{\prime}\right\}_{n}$ converges uniformly on $[a, b]$ to $g$ " is replaced by " $\left\{f_{n}^{\prime}\right\}_{n}$ converges pointwise on $[a, b]$ to $g$ ", then, even if $\left\{f_{n}\right\}_{n}$ converges uniformly to $f$ on $[a, b]$, it may happen that $f^{\prime}$ exists and 
is continuous on $[a, b]$, but $f^{\prime} \neq g$ on a perfect set of positive measure as close as we want to $b-a$ (see Example 2).

If in Theorem B the condition " $\left\{\left(f_{n}\right)_{a p}^{\prime}\right\}_{n}$ converges uniformly on $[a, b]$ to $g$ " is replaced by " $\left\{\left(f_{n}\right)_{a p}^{\prime}\right\}_{n}$ converges pointwise on $[a, b]$ to $g$ ", then, even if $\left\{f_{n}\right\}_{n}$ converges uniformly to $f$ on $[a, b]$, it may happen that $f_{a p}^{\prime}$ exists and is continuous on $[a, b]$, but $f_{a p}^{\prime} \neq g$ on a perfect set of positive measure as close as we want to $b-a$ (see Example 2).

\section{$5 \quad$ Examples}

Example 1. First we construct a Cantor type perfect set, contained in $[0,1]$. Let $\beta \in(0,1]$ and let $\left\{\beta_{n}\right\}_{n}$ be a sequence of positive numbers such that $\sum_{n=1}^{\infty} 2^{n-1} \beta_{n}=\beta$. We extract from $[0,1]$ the open interval $G_{1}=\left(a_{1}, b_{1}\right)$, centered in $1 / 2$ with length $\beta_{1}$.

Let

$$
P_{1}=[0,1] \backslash G_{1} .
$$

Clearly $P_{1}$ consists of two disjoint closed intervals, each of length

$$
\frac{1-\beta_{1}}{2} \text {. }
$$

From each of the two intervals of $P_{1}$ we extract from the left to the right the centered open intervals $\left(a_{2}, b_{2}\right)$ and $\left(a_{3}, b_{3}\right)$, with length $\beta_{2}$. Let

$$
G_{2}=G_{1} \cup\left(a_{2}, b_{2}\right) \cup\left(a_{3}, b_{3}\right) \text { and } P_{2}=[0,1] \backslash G_{2} \text {. }
$$

Clearly $P_{2}$ consists of $2^{2}$ nonoverlapping closed intervals, each of length

$$
\frac{1-\left(\beta_{1}+2 \beta_{2}\right)}{2^{2}}
$$

Suppose we have already defined the sets $G_{n-1}$ and $P_{n-1}, n \geq 2$. Then $P_{n-1}$ consists of $2^{n-1}$ nonoverlapping closed intervals, each of length

$$
\frac{1-\left(\beta_{1}+2 \beta_{2}+\cdots+2^{n-1} \beta_{n-1}\right)}{2^{n-1}} .
$$

From each interval of $P_{n-1}$ we extract from the left to the right the centered open intervals

$$
\left(a_{2^{n-1}}, b_{2^{n-1}}\right),\left(a_{2^{n-1}+1}, b_{2^{n-1}+1}\right), \ldots,\left(a_{2^{n}-1}, b_{2^{n}-1}\right)
$$


with length $\beta_{n}$. Let

$$
G_{n}=G_{n-1} \cup\left(\cup_{i=2^{n-1}}^{2^{n}-1}\left(a_{i}, b_{i}\right)\right) \text { and } P_{n}=[0,1] \backslash G_{n} .
$$

Then $P_{n}$ consists of $2^{n}$ nonoverlapping closed intervals, each of length

$$
\frac{1-\left(\beta_{1}+2 \beta_{2}+\cdots+2^{n} \beta_{n}\right)}{2^{n}} .
$$

Let

$$
G=\cup_{n=1}^{\infty} G_{n} \text { and } P=\cap_{n=1}^{\infty} P_{n} .
$$

Then $m(G)=\beta$ and $m(P)=1-\beta$.

Let $f:[0,1] \rightarrow \mathbb{R}$,

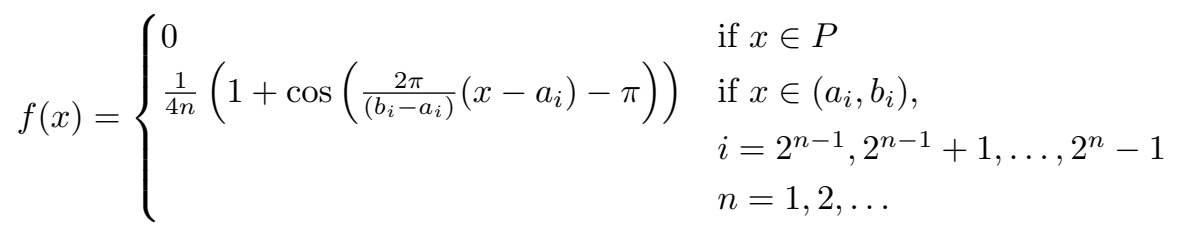

Let $f_{n}:[0,1] \rightarrow \mathbb{R}$ (for $f_{3}$ see Figure 1$), f_{n}(x)= \begin{cases}f(x) & \text { if } x \in G_{n} \\ 0 & \text { if } x \in P_{n}\end{cases}$

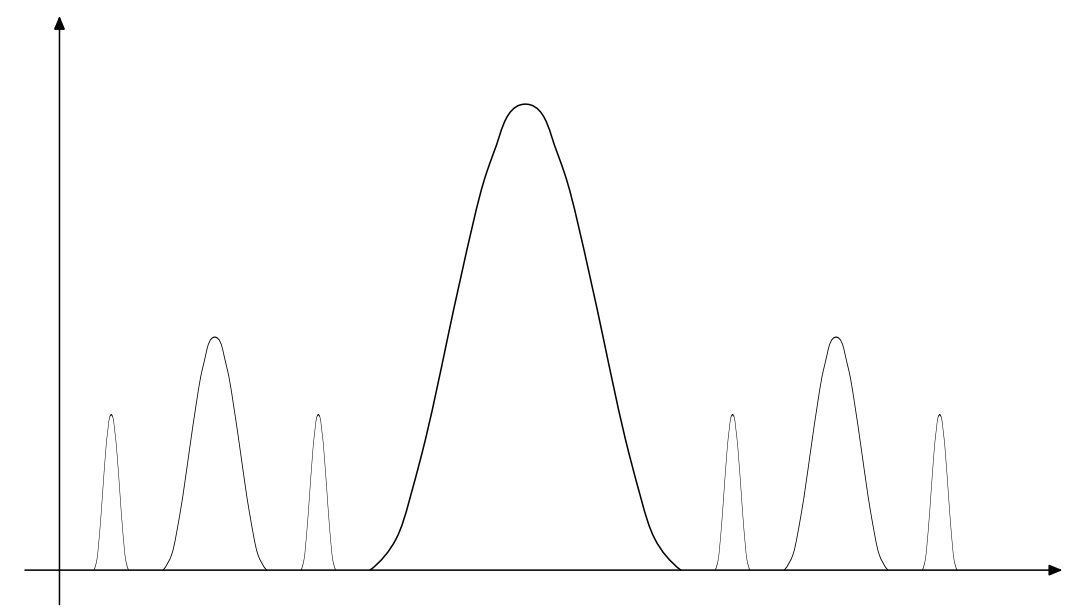

Figure 1: The graph of $f_{3}$ in Example 1 
Then we have

1) $f_{n}^{\prime}(x)=\left\{\begin{array}{ll}0 & \text { if } x \in P_{n} \\ f^{\prime}(x) & \text { if } x \in G_{n}, n=1,2, \ldots\end{array}\right.$ hence $\left\{f_{n}\right\}_{n} \in C^{1}([0,1])$;

2) $f_{n} \longrightarrow f[$ unif $]$ on $[0,1]$;

3) Let $g:[0,1] \rightarrow \mathbb{R}, g(x)= \begin{cases}0 & x \in P \\ f^{\prime}(x) & x \in G .\end{cases}$

Then $f_{n}^{\prime}(x) \rightarrow g(x),(\forall) x \in[0,1]$.

4) $f^{\prime}(x)$ does not exist (finite or infinite) if $x \in P$, but $f_{a p}^{\prime}=g$ a.e. on $[0,1]$.

5) $\left\{f_{n}\right\}_{n}$ is $U A C$ on $P$, but $\left\{f_{n}\right\}_{n}$ is not $U A C^{*}$ (and neither $U A C^{*} G$ ) on $P$ (see Corollary 4, (II) and Theorem 1, (ii)).

Example 2. We consider all the notations of Example 1. Let $\left\{\alpha_{n}\right\}$ be a strictly increasing sequence of positive numbers, converging to 1 . From each $\left(a_{i}, b_{i}\right), i=1,2, \ldots, 2^{n}-1$, we extract the centered closed interval $\left[c_{i}^{n}, d_{i}^{n}\right]$ of length $\alpha_{n}\left(b_{i}-a_{i}\right)$. Let

$$
K_{n}=\cup_{i=1}^{2^{n}-1}\left[c_{i}^{n}, d_{i}^{n}\right] .
$$

Then $m\left(K_{n}\right)=\alpha_{n} \cdot m\left(G_{n}\right)$ and $G=\cup_{n=1}^{\infty} K_{n}$. Let $f_{n}:[0,1] \rightarrow \mathbb{R}, n=1,2, \ldots$ First we define $f_{n}$ on $P_{n} \cup K_{n}$ by

$$
f_{n}(x)= \begin{cases}\alpha & \text { if } x \in[\alpha, \beta] \\ x-\frac{m\left(P_{n}\right)}{2^{n+1}} & \text { if } x \in K_{n},\end{cases}
$$

where $[\alpha, \beta]$ is any of the $2^{n}$ closed intervals of $P_{n}$. Clearly $f_{n}$ is increasing on $P_{n} \cup K_{n}$. On each $\left[a_{i}, c_{i}^{n}\right], i=1,2, \ldots, 2^{n}-1$, we define $f_{n}$ such that $f_{n}$ is strictly increasing, $f_{n}$ has a continuous derivative on $\left[a_{i}, c_{i}^{n}\right], f_{n}^{\prime}\left(a_{i}\right)=0$ and $f_{n}^{\prime}\left(c_{i}^{n}\right)=1$. On each $\left[d_{i}^{n}, b_{i}\right], i=1,2, \ldots, 2^{n}-1$, we define $f_{n}$ such that $f_{n}$ is strictly increasing, $f_{n}$ has a continuous derivative on $\left[d_{i}^{n}, b_{i}\right], f^{\prime}\left(d_{i}^{n}\right)=1$ and $f_{n}^{\prime}\left(b_{i}\right)=0$ (for $f_{3}$ and $f$ see Figure 2 ).

Then we have

1) $f_{n} \in C^{1}([0,1])$;

2) $f_{n} \longrightarrow f[$ unif $]$ on $[0,1]$, where $f:[0,1] \rightarrow[0,1], f(x)=x, f \in C^{1}[0,1]$;

3) $f_{n}^{\prime}(x)=0$ on $P_{n}$. Hence $f_{n}^{\prime}(x)=0$ on $P$. 
4) $\lim _{n \rightarrow \infty} f_{n}^{\prime}(x)=1$ for each $x \in G$. (Indeed, for $x \in G=\cup_{n=1}^{\infty} K_{n}$, there exists a positive integer $m$ such that $x \in \operatorname{int}\left(K_{n}\right),(\forall) n \geq m$, because $K_{1} \subset K_{2} \subset \ldots \subset K_{m} \subset \ldots \subset K_{n} \subset \ldots$; it follows that $f_{n}^{\prime}(x)=1$, $(\forall) n \geq m$, so $\left.\lim _{n \rightarrow \infty} f_{n}^{\prime}(x)=1\right)$.

5) $\lim _{n \rightarrow \infty} f_{n}^{\prime}(x)=g(x), x \in[0,1]$, where $g:[0,1] \rightarrow[0,1]$,

$$
g(x)= \begin{cases}0 & \text { if } x \in P \\ 1 & \text { if } x \in G\end{cases}
$$

6) $\left\{f_{n}\right\}_{n}$ is $U A C$ (or $U A C G$ ) neither on $[0,1]$ nor on $P$ (see Corollary 4 , (I) and Theorem 1, (i)).

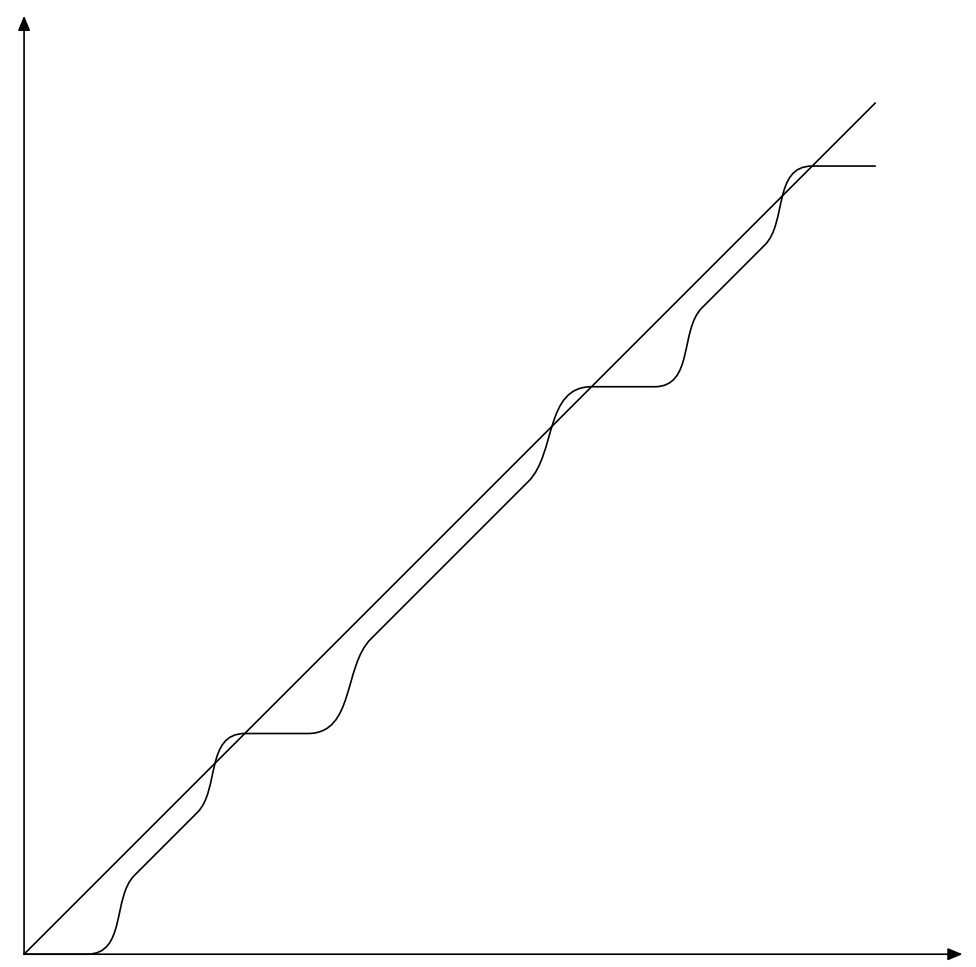

Figure 2: The graph of $f_{3}$ and $f$ in Example 2 


\section{References}

[1] A. M. Bruckner, Differentiation of real functions, Lect. Notes in Math., vol. 659, Springer-Verlag, 1978.

[2] A. M. Bruckner and C. Goffman, Approximate differentiation, Real Analysis Exchange 6 (1980-81), 9-65.

[3] V. G. Čelidze and A. G. Džvaršeišvili, The theory of the Denjoy integral and some applications, World Scientific, 1978.

[4] V. Ene, Real functions - current topics, Lect. Notes in Math., vol. 1603, Springer-Verlag, 1995.

[5] Y. Kubota, An integral of Denjoy type, Proc. Japan Acad 40 (1964), $713-717$.

[6] C. M. Lee, An analogue of the theorem Hake-Alexandroff-Looman, Fund. Math. C (1978), 69-74.

[7] P. Y. Lee, Lanzhou lectures on Henstock integration, World Scientific, Singapore, 1989.

[8] I. P. Natanson, Theory of functions of a real variable, 2nd. rev. ed., Ungar, New York, 1961.

[9] J. Ridder, Über die gegenseitigen Beziehungen verschiedener approximativ stetigen Denjoy-Perron Integrale, Fund. Math. 22 (1934), 136-162.

[10] W. Rudin, Principles of mathematical analysis, 2nd. ed., McGraw-Hill Book Company, New York, San Francisco, Toronto, London, 1964.

[11] S. Saks, Theory of the integral, 2nd. rev. ed., vol. PWN, Monografie Matematyczne, Warsaw, 1937.

[12] B. S. Thomson, Real functions, Lect. Notes in Math., vol. 1170, SpringerVerlag, 1985. 